\title{
Electromagnetic Radiation from Arbitrarily Shaped Microstrip Antenna Using the Equivalent Dipole-Moment Method
}

\author{
Jiade Yuan and Kaixiong Su \\ College of Physics and Information Engineering, Fuzhou University, Fujian, Fuzhou 350108, China \\ Correspondence should be addressed to Jiade Yuan, yuanjd2010@yahoo.com.cn
}

Received 26 December 2011; Revised 23 February 2012; Accepted 23 February 2012

Academic Editor: Hon Tat Hui

Copyright () 2012 J. Yuan and K. Su. This is an open access article distributed under the Creative Commons Attribution License, which permits unrestricted use, distribution, and reproduction in any medium, provided the original work is properly cited.

The equivalent dipole-moment method (EDM) is extended and applied in the analysis of electromagnetic (EM) radiation by arbitrarily shaped microstrip antenna in this paper. The method of moments (MoM) is used to solve the volume-surface integral equation (VSIE). A strip model is applied in the treatment of the feeding probe of the microstrip antenna, in which the discretized triangular elements of the excitation source are equivalent as dipole models. The proposed approach is sufficiently versatile in handling arbitrarily shaped microstrip antenna and is easily constructed through a simple procedure. Numerical results are given to demonstrate the accuracy and efficiency of this method.

\section{Introduction}

Conformal microstrip structures have received increasing attention in the last decades, due to the need of antennas and devices that can be mounted on curved surfaces. Analysis and design of conformal microstrip antenna are more complicated than planar microstrip antenna [1]. The method of moments (MoM) [2] is commonly used in the full-wave methods owing to its accuracy. A number of the method of moments (MoM)-based full-wave solutions have been proposed to analyze the microstrip antennas with various shapes [3-6]. Among them, the volume-surface integral equation (VSIE) method $[5,6]$ is one of the most attractive approaches due to its flexibility and easy conjunction. However, the conventional VSIE approache to computing the MoM impedance matrix elements consumes a considerable portion of the total solution time, and this, in turn, can place an inordinately heavy burden on the CPU in terms of memory and time as the problem dimensions become large in terms of the wavelength. Fortunately, several efficient VSIE-based methods have been studied in recent years such as the fast multipole method (FMM) and the multilevel FMM [7], the precorrected-FFT method [5], the integral equation fast Fourier transform algorithm [8], and the characteristic basis function method [9]. These methods play a great role in saving CPU time or reducing the memory requirement to some extent.

More recently, the equivalent dipole-moment method $[10,11]$ has been proposed to efficiently analyze the electromagnetic scattering by the composite metallic and dielectric objects. In this paper, the equivalent dipole-moment method is extended to analyze the EM radiation problems of arbitrary shaped microstrip antenna. From the scheme and formulation, we can find that though the problem becomes much more complicated for the arbitrary shaped microstrip antenna, each impedance matrix element can still be expressed in a unified simple form. The article is organized as follows. Section 2 introduces the theory of the EDM and its voltage model in detail, and Section 3 shows the numerical results. The final section presents the conclusions.

\section{Theory}

In this section, we present the theoretical foundation of the procedure for efficient generation of MoM matrices associated with the coupled VSIE. The detailed derivation of the VSIE and the definition of RWG $\left(\mathbf{f}_{s m}\right)$ and SWG $\left(\mathbf{f}_{v m}\right)$ basis functions can be found in [12-14]. The VSIE [14] can 
be expressed as

$$
\begin{gathered}
\sum_{n=1}^{N_{s}} I_{s n} Z_{m n}^{s s}+\sum_{n=1}^{N_{v}} I_{v n} Z_{m n}^{s v}=\left\langle\mathbf{E}^{i}, \mathbf{f}_{s m}\right\rangle, \\
\sum_{n=1}^{N_{s}} I_{s n} Z_{m n}^{v s}+\sum_{n=1}^{N_{v}} I_{v n} Z_{m n}^{v v}=\left\langle\mathbf{E}^{i}, \mathbf{f}_{v m}\right\rangle \quad m=1,2, \ldots, N,
\end{gathered}
$$

where

$$
\begin{aligned}
& Z_{m n}^{s s}=-\left\langle\mathbf{E}_{s n}^{s}, \mathbf{f}_{s m}(\mathbf{r})\right\rangle, \\
& Z_{m n}^{s v}=-\left\langle\mathbf{E}_{v n}^{s}, \mathbf{f}_{s m}(\mathbf{r})\right\rangle, \\
& Z_{m n}^{v s}=-\left\langle\mathbf{E}_{s n}^{s}, \mathbf{f}_{v m}(\mathbf{r})\right\rangle, \\
& Z_{m n}^{v v}=\left\langle\mathbf{f}_{v n}(\mathbf{r}) /\left(j \omega \varepsilon(\mathbf{r}), \mathbf{f}_{v m}(\mathbf{r})\right\rangle-\left\langle\mathbf{E}_{v n}^{s}, \mathbf{f}_{v m}(\mathbf{r})\right\rangle,\right.
\end{aligned}
$$

where the " $\langle\cdots\rangle$ " is the mathematical symbol of interproduct, $Z_{m n}^{t u}(t=s, v, u=s, v)$ stands for the impedance matrix element in row $m$ and column $n$. $\mathbf{E}_{u n}^{s}(u=s, v)$ stands for the radiated electric field from the equivalent current of the $n$th RWG or SWG element.

On the conducting surface S, a RWG [12] element $n$ with two triangles $T_{n}^{ \pm}$adjacent to the edge of length $l_{n}$ is shown in Figure $1(\mathrm{a})$. The $n$th dipole moment $\mathbf{m}_{s}$ is obtained by the integration of the surface current, corresponding to edge element $n$, over the element surface

$$
\mathbf{m}_{s}=\int_{T_{n}^{+}+T_{n}^{-}} \mathbf{J}_{s n}\left(\mathbf{r}^{\prime}\right) d s^{\prime}=I_{s n} \mathbf{m}_{\mathrm{sn}},
$$

where

$$
\mathbf{m}_{s n} \approx l_{n}\left(\mathbf{r}_{n}^{c-}-\mathbf{r}_{n}^{c+}\right),
$$

In the dielectric body $V$, described by a SWG [13] element $n$ with two inner tetrahedrons $T_{n}^{ \pm}$adjacent to the common face of area $a_{n}$ is shown in Figure 1(b). If the $n$th face is a boundary face, which is associated with only one tetrahedron, this corresponding tetrahedron is always assumed as $T_{n}^{+}$, as shown in Figure $1(\mathrm{c})$. The $n$th dipole moment $\mathbf{m}_{v}$ is obtained by the integration of the volume current, corresponding to face element $n$, over the element volume

$$
\mathbf{m}_{v}=\int_{T_{n}^{+}+T_{n}^{-}} \mathbf{J}_{v n}\left(\mathbf{r}^{\prime}\right) d v^{\prime}=I_{v n} \mathbf{m}_{v n}
$$

where

$$
\mathbf{m}_{v n} \approx \begin{cases}a_{n} \kappa(\mathbf{r})\left(\mathbf{r}_{n}^{c-}-\mathbf{r}_{n}^{c+}\right) & T_{n}^{ \pm} \in V, \\ a_{n} \kappa(\mathbf{r})\left(\mathbf{r}_{n s}^{c}-\mathbf{r}_{n}^{c+}\right) & T_{n}^{+} \in V, T_{n}^{-} \notin V,\end{cases}
$$

where the dielectric material of each dipole model is assumed uniform. The $\mathbf{r}_{n}^{c-}, \mathbf{r}_{n}^{c+}$ and $\mathbf{r}_{n s}^{c}$ are the centroid radius vectors of $T_{n}^{+}, T_{n}^{-}$and the $n$th boundary face, respectively, as shown in Figure 1.
Referring to [10], the radiated electric fields of the $n$th infinitesimal dipole at the field point $\mathbf{r}$ can be expressed as

$$
\begin{array}{r}
\mathbf{E}_{u n}^{s}(\mathbf{r})=\frac{\eta}{4 \pi}\left[\left(\mathbf{M}_{u n}-\mathbf{m}_{u n}\right)\left(\frac{j k}{R}+C\right)+2 \mathbf{M}_{u n} C\right] e^{-j k R}, \\
u=s, v
\end{array}
$$

in which

$$
\begin{gathered}
C=\frac{1}{R^{2}}\left[1+\frac{1}{j k R}\right], \\
\mathbf{M}_{u}=\frac{\left(\mathbf{R} \cdot \mathbf{m}_{u}\right) \mathbf{R}}{R^{2}},
\end{gathered}
$$

where $\mathbf{R}=\mathbf{r}-\mathbf{r}^{\prime}$ and $R=|\mathbf{R}|$. Equation (10) is the exact expressions and valid at arbitrary distances from the dipole, not only in the far field. However, if the observation point is close to the dipole for a separation distance of less than a threshold distance between the source and the testing function locations, the SWG or RWG element can not be regarded as an infinitesimal dipole model, so that (10) will not hold. In this case, the conventional MoM is still used to calculate the impedance matrix element, otherwise the MoM matrix elements are computed by the EDM method directly.

Substituting (10) into (2)-(5) and referring to $[12,13]$, the expressions of the impedance matrix element $Z_{m, n}$ are calculated by

$$
\mathrm{Z}_{m, n} \approx\left\{\begin{array}{rr}
-l_{m}\left[\left.e^{-j k R} \mathbf{E}_{u n}^{s}(R)\right|_{R=\left|\mathbf{r}_{m}^{\prime}-\mathbf{r}_{n}^{\prime}\right|} \cdot\left(\mathbf{r}_{m}^{c-}-\mathbf{r}_{m}^{c+}\right)\right] & T_{m}^{ \pm} \in S, \\
-a_{m}\left[\left.e^{-j k R} \mathbf{E}_{u n}^{s}(R)\right|_{R=\left|\mathbf{r}_{m}^{\prime}-\mathbf{r}_{n}^{\prime}\right|} \cdot\left(\mathbf{r}_{m}^{c-}-\mathbf{r}_{m}^{c+}\right)\right] & T_{m}^{ \pm} \in V, \\
-a_{m}\left[\left.e^{-j k R} \mathbf{E}_{u n}^{s}(R)\right|_{R=\left|\mathbf{r}_{m}^{\prime}-\mathbf{r}_{n}^{\prime}\right|} \cdot\left(\mathbf{r}_{m s}^{c}-\mathbf{r}_{m}^{c+}\right)\right] \\
T_{m}^{+} \in V, T_{m}^{-} \notin V,
\end{array}\right.
$$

where the $\mathbf{r}_{m}^{c-}, \mathbf{r}_{m}^{c+}$, and $\mathbf{r}_{m s}^{c}$ are the centroid radius vectors of $T_{m}^{+}, T_{m}^{-}$, and the $m$ th face, respectively. $\mathbf{r}_{n}^{\prime}$ and $\mathbf{r}_{m}^{\prime}$ are the centre radius vector of the $n$th and the $m$ th equivalent dipole model respectively,

$$
\mathbf{r}_{u}^{\prime}=\left\{\begin{array}{ll}
\frac{\left(\mathbf{r}_{u}^{c+}+\mathbf{r}_{u}^{c-}\right)}{2} & T_{u}^{ \pm} \in V, S, \\
\frac{\left(\mathbf{r}_{u}^{c+}+\mathbf{r}_{u s}^{c}\right)}{2} & T_{u}^{+} \in V, T_{u}^{-} \notin V .
\end{array} \quad u=m, n\right.
$$

A simple equivalent strip model [6] is applied in the treatment of the feeding probe, where the wire-surface junction is changed to a surface-surface junction. The feeding probe of diameter $d$ is replaced with its equivalent strip structure with the width $w=2 d$. There are 3 triangles, marked as $1,2,3$, sharing the surface-surface junction, of $l_{n}$, 


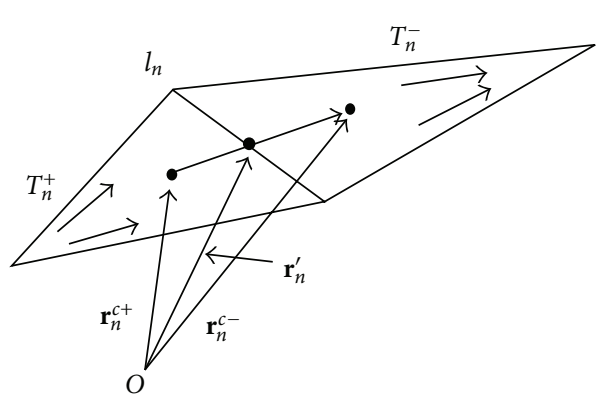

(a)

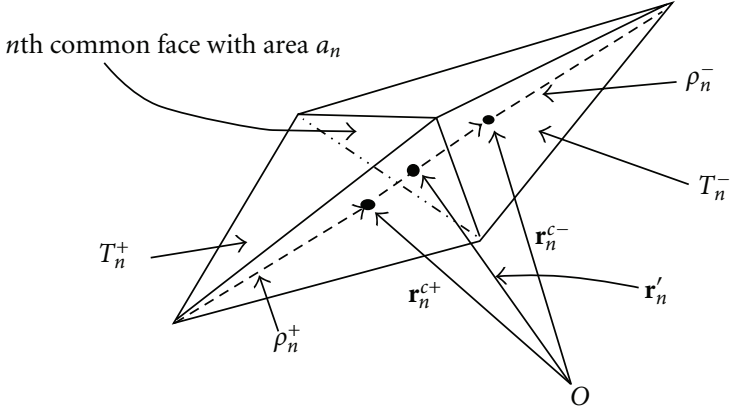

(b)

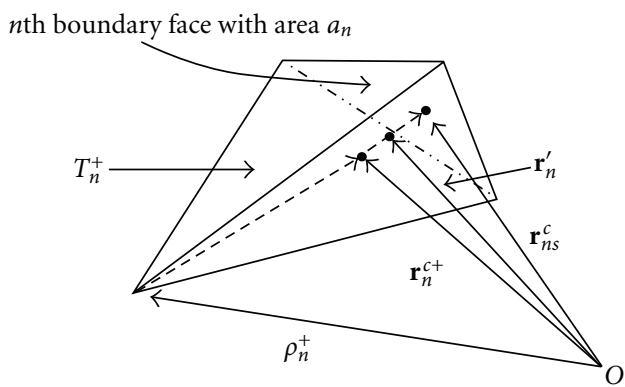

(c)

Figure 1: (a) Pair of triangle patches with the $n$th common edge $l_{n}$. (b) Pair of tetrahedrons associated with the $n$th common face. (c) The $n$th boundary face associated with one tetrahedron.

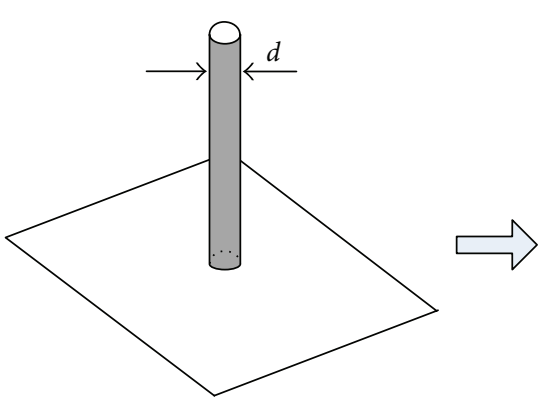

(a)

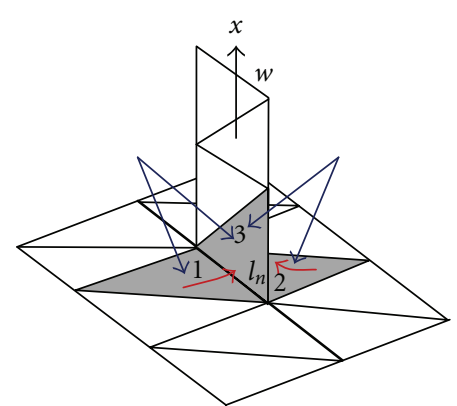

(b)

Figure 2: Dipole model in the excitation source of antenna. (a) Wire-surface junction. (b) Surface-surface junction and its equivalent electric dipole model.

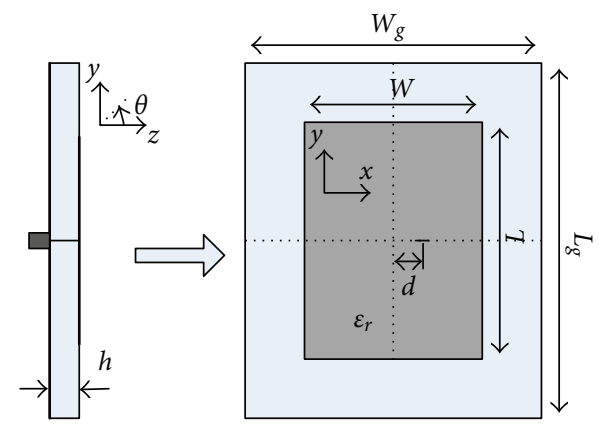

Figure 3: Geometry of a probe feed microstrip antenna. 


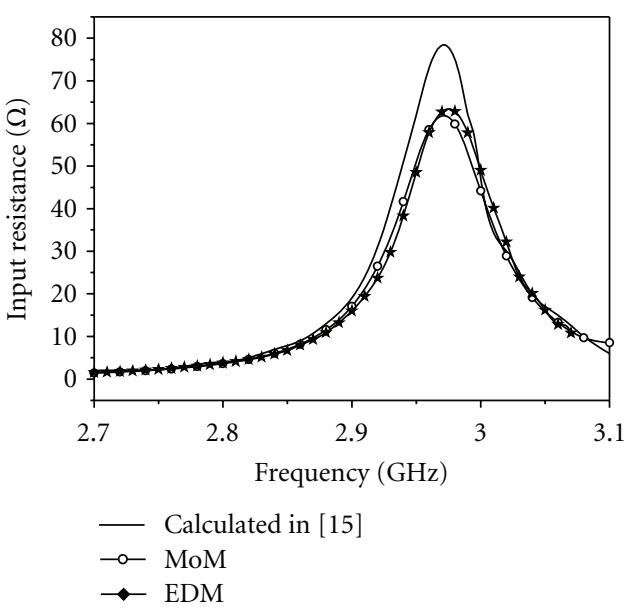

(a)

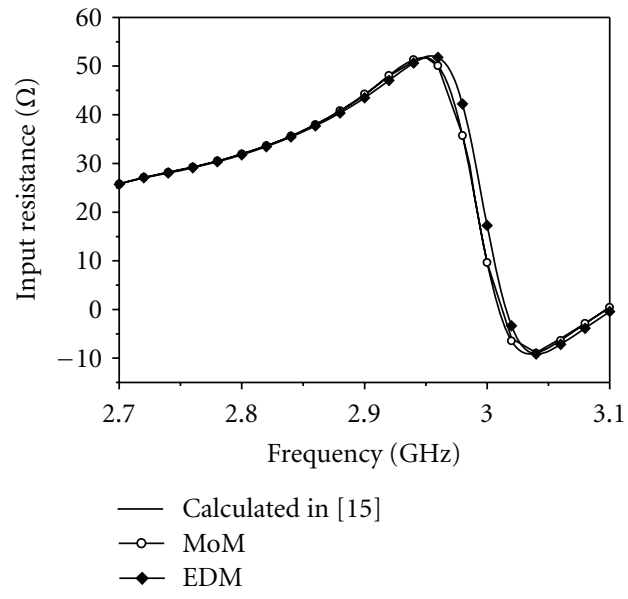

(b)

FIGURE 4: Input impedance of the planar microstrip antenna. (a) Input resistance. (b) Input reactance.

which are viewed as 2 RWG basis functions, that is, $(1,3)$, $(2,3)$. In EDM, the two triangle pairs, $(1,3)$ and $(2,3)$, are viewed as the equivalent electric dipole model, respectively, as shown in Figure 2. The excitation source locates at the junction and the $\mathbf{E}^{i}$ in (1) can be established by using the delta-gap voltage model [10]

$$
\mathbf{E}^{i}=V \delta(l) \mathbf{n}_{l},
$$

where $V$ is the voltage across the gap, and $\mathbf{n}_{l}$ is normal to the junction $l_{n}$.

\section{Numerical Results}

In this section, some numerical results are presented to validate the algorithm, and the efficiency of the method is demonstrated through comparison.

To verify the correctness and applicability of our theory as well as the program code, we first consider a plane microstrip antenna on a finite substrate. Figure 3 shows the structure of the antenna. The size of the substrate is $\mathrm{W}_{g} \times$ $L_{g}=50 \mathrm{~mm} \times 60 \mathrm{~mm}$, while the size of the patch is $\mathrm{W} \times L=$ $30 \mathrm{~mm} \times 40 \mathrm{~mm}$, respectively. The radius of the feed probe is $0.75 \mathrm{~mm}$. The feeding point is on the centerline of the Lside and is $d=6.5 \mathrm{~mm}$ from the center of the patch. The substrate's thickness is $h=1.5 \mathrm{~mm}$ with a dielectric constant of $\varepsilon_{r}=2.55$.

The mesh of the equivalent strip model should be refined, and the maximum edge length of triangles on strip model and metal surface is chosen as less than $\lambda_{0} / 20\left(\lambda_{0}\right.$ is the wavelength in free space) on the excitation source of antenna, while $\lambda_{g} / 15$ ( $\lambda_{g}$ is the wavelength in dielectric) is chosen as the maximum edge length for the tetrahedral cells, respectively. Meanwhile, $0.15 \lambda_{0}$ is chosen in EDM as the threshold distance for both the metal surface and dielectric volume. The conventional MoM and the EDM-accelerated MoM are applied to compute the input impedance and compared with the results given in [15]. Figure 4 shows the input resistance and the input reactance from 2.7 to $3.1 \mathrm{GHz}$, respectively, and good agreement is observed.

In the computation, the microstrip antenna is discretized into 502 triangular patches and the 1031 tetrahedrons, yielding a total number of 3163 unknowns. The number of the calculated frequency points is chosen 20 , and the total CPU time is 5223 seconds in EDM method, and 7142 seconds in conventional method respectively, which yields a reduction of $26.9 \%$.

In second example, we consider a cylindrically conformal microstrip patch antennas; as shown in Figure 5 the length of the patch is $L \times W=30 \mathrm{~mm} \times 40 \mathrm{~mm}$, while the sizes of the substrate are $L_{g} \times W_{g}=50 \mathrm{~mm} \times 60 \mathrm{~mm}$, the inner radius of the curvature of the substrate is $50 \mathrm{~mm}$, and the feeding point is on the centerline of the curved side and is $10 \mathrm{~mm}$ from the center of the patch. The thickness of the substrate is $h=0.795 \mathrm{~mm}$. The permittivity of the substrate is $\varepsilon_{r}=2.32$.

The maximum size of mesh discretization and the threshold distance in EDM are the same as the first numerical example. The EDM is applied to compute the return loss and compared with the results from the conventional MoM. Figure 6 shows the return loss from 2.9 to $3.5 \mathrm{GHz}$. It is observed that the results of the EDM are in better agreement with the conventional MoM.

In the computation, the conformal microstrip antenna is discretized into 400 triangular patches and the 1340 tetrahedrons, yielding a total number of 4035 unknowns. The number of the calculated frequency points is chosen 30 , and the total CPU time is 11967 seconds in EDM method and 17364 seconds in conventional method, respectively, which yields a reduction of $31.1 \%$.

\section{Conclusion}

The EDM method has been applied to analyze the EM radiation characteristics of arbitrarily shaped microstrip antenna on finite-sized substrate. The antennas are discretized into tetrahedral volume elements in the material region and 


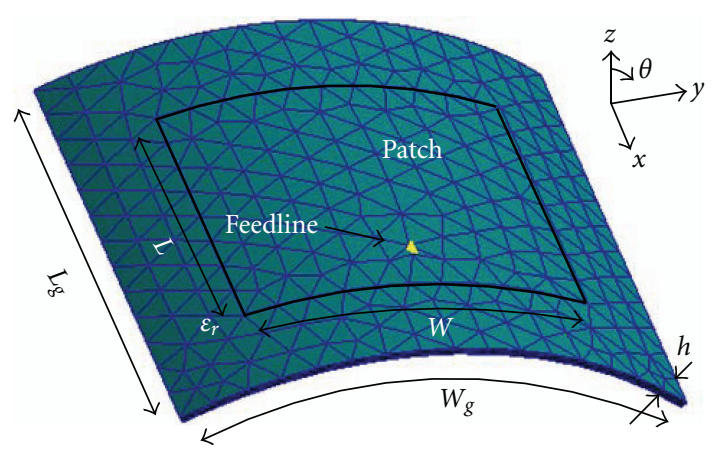

FIGURE 5: Geometry of a conformal microstrip antenna.

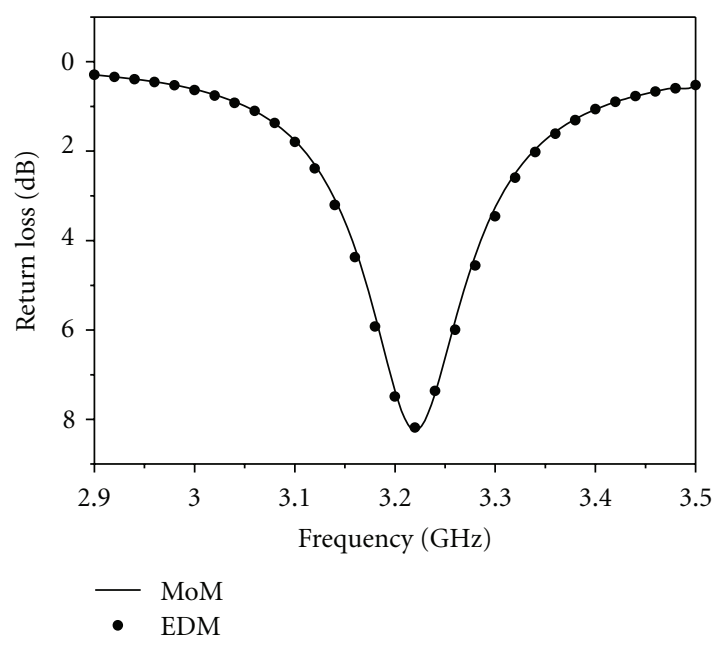

FIgURE 6: Ruturn loss as a function of frequency.

into triangle patches on the conducting surface, where the volume-surface integral equation (VSIE) is setup. Then the method of moments (MoM) is employed to solve the VSIE. A simple equivalent strip model has been established and the EDM has been applied in the treatment of the excitation source of the microstrip antenna, where the surface-surface junction associated with three triangles is equivalent as two dipole models. Numerical results validate the accuracy of the equivalent treatment method. The application of the EDM method significantly reduces the complexity of the computation to the impedance matrix as well as the solution time. Even when the problems become more complicated for the introduction of the probe-fed model in the conventional MoM, the expressions of impedance matrix element in the EDM method still keep a simple form and are very easy constructed by using a simple procedure.

\section{Acknowledgments}

This work was supported by Natural Science Foundation of Fujian Province of China (2011J01348) and the Foundation of Fuzhou University (XRC-1057).

\section{References}

[1] L. Josefsson and P. Persson, Conformal Array Antenna Theory and Design, IEEE Press; John Wiley \& Sons, New York, NY, USA, 2006.

[2] R. F. Harrington, Field Computation by Moment Methods, Wiley-IEEE Press, NewYork, NY, USA, 1993.

[3] S. K. Khamas, "A generalized asymptotic extraction solution for antennas in multilayered spherical media," IEEE Transactions on Antennas and Propagation, vol. 58, no. 11, pp. 37433747, 2010.

[4] V. B. Ertürk and R. G. Rojas, "Efficient analysis of input impedance and mutual coupling of microstrip antennas mounted on large coated cylinders," IEEE Transactions on Antennas and Propagation, vol. 51, no. 4, pp. 739-749, 2003.

[5] N. Yuan, T. Soon Yeo, X.-C. Nie et al., "Analysis of probe-fed conformal microstrip antennas on finite grounded substrate," IEEE Transactions on Antennas and Propagation, vol. 54, no. 2, pp. 554-563, 2006.

[6] M. He, Q. Chen, Q. Yuan, K. Sawaya, and X. Xu, "A simple strip model in the volume-surface integral equation for analysis of arbitrary probe-fed conformal microstrip antennas," IEEE Antennas and Wireless Propagation Letters, vol. 8, pp. 530-533, 2009.

[7] D. Ding, S. Tao, Z. Fan, and R. Chen, "Fast analysis of finite and curved frequency selective surfaces using the VSIE with MLFMA," in Proceedings of the IEEE International Conference on Ultra-Wideband (ICUWB '10), pp. 364-367, September 2010.

[8] J. Yin, J. Hu, and Z. Nie, "IE-FFT solution of volumesurface integral equation for composite conducting-dielectric objects," in Proceedings of the International Conference on Microwave Technology and Computational Electromagnetics, pp. 414-417, 2009.

[9] J. Yuan, C. Gu, and G. Han, "A hybrid equivalent dipole moment and adaptive modified characteristic basis function method for electromagnetic scattering by multilayered dielectric bodies," International Journal of RF and Microwave Computer-Aided Engineering, vol. 19, no. 6, pp. 685-691, 2009.

[10] J. Yuan, C. Gu, and G. Han, "Efficient generation of method of moments matrices using equivalent dipole-moment method," IEEE Antennas and Wireless Propagation Letters, vol. 8, pp. 716-719, 2009.

[11] J. Yuan, Z. Niu, Z. Li, and C. Gu, "Electromagnetic scattering by arbitrarily shaped PEC targets coated with anisotropic media using equivalent dipole-moment method," Journal of Infrared, Millimeter, and Terahertz Waves, vol. 31, no. 6, pp. 744-752, 2010.

[12] S. M. Rao, D. R. Wilton, and A. W. Glisson, "Electromagnetic scattering by surfaces of arbitrary shape," IEEE Transactions on Antennas and Propagation, vol. 30, no. 3, pp. 409-418, 1982.

[13] D. H. Schaubert, D. R. Wilton, and A. W. Glisson, "A tetrahedral modeling method for electromagnetic scattering by arbitrarily shaped inhomogeneous dielectric bodies," IEEE Transactions on Antennas and Propagation, vol. 32, no. 1, pp. 77-85, 1984.

[14] X. C. Nie, N. Yuan, L. W. Li, Y. B. Gan, and T. S. Yeo, "A fast volume-surface integral equation solver for scattering from composite conducting-dielectric objects," IEEE Transactions on Antennas and Propagation, vol. 53, no. 2, pp. 818-824, 2005.

[15] S. N. Makarov, S. D. Kulkarni, A. G. Marut, and L. C. Kempel, "Method of moments solution for a printed patch/slot antenna on a thin finite dielectric substrate using the volume integral equation," IEEE Transactions on Antennas and Propagation, vol. 54, no. 4, pp. 1174-1184, 2006. 

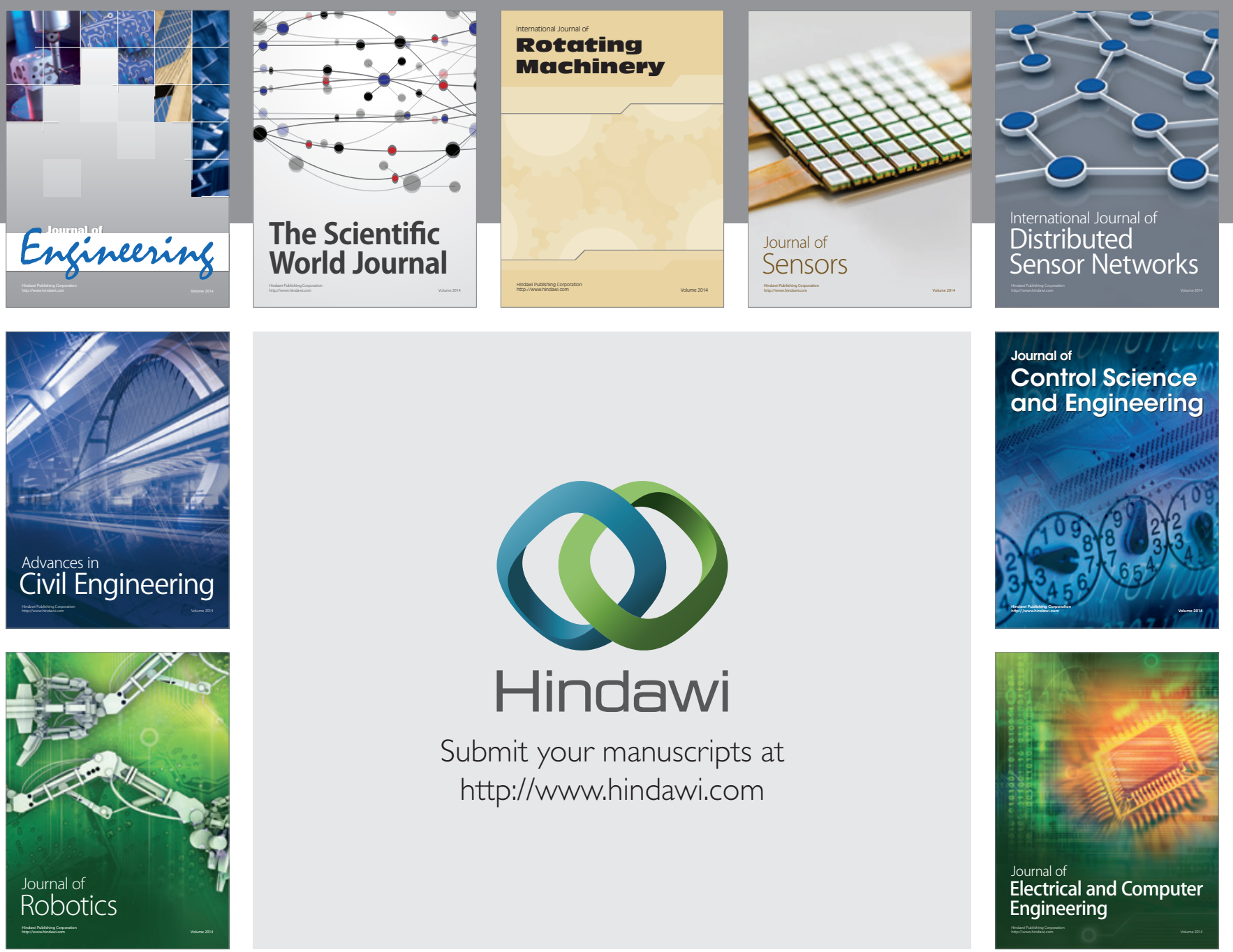

Submit your manuscripts at

http://www.hindawi.com
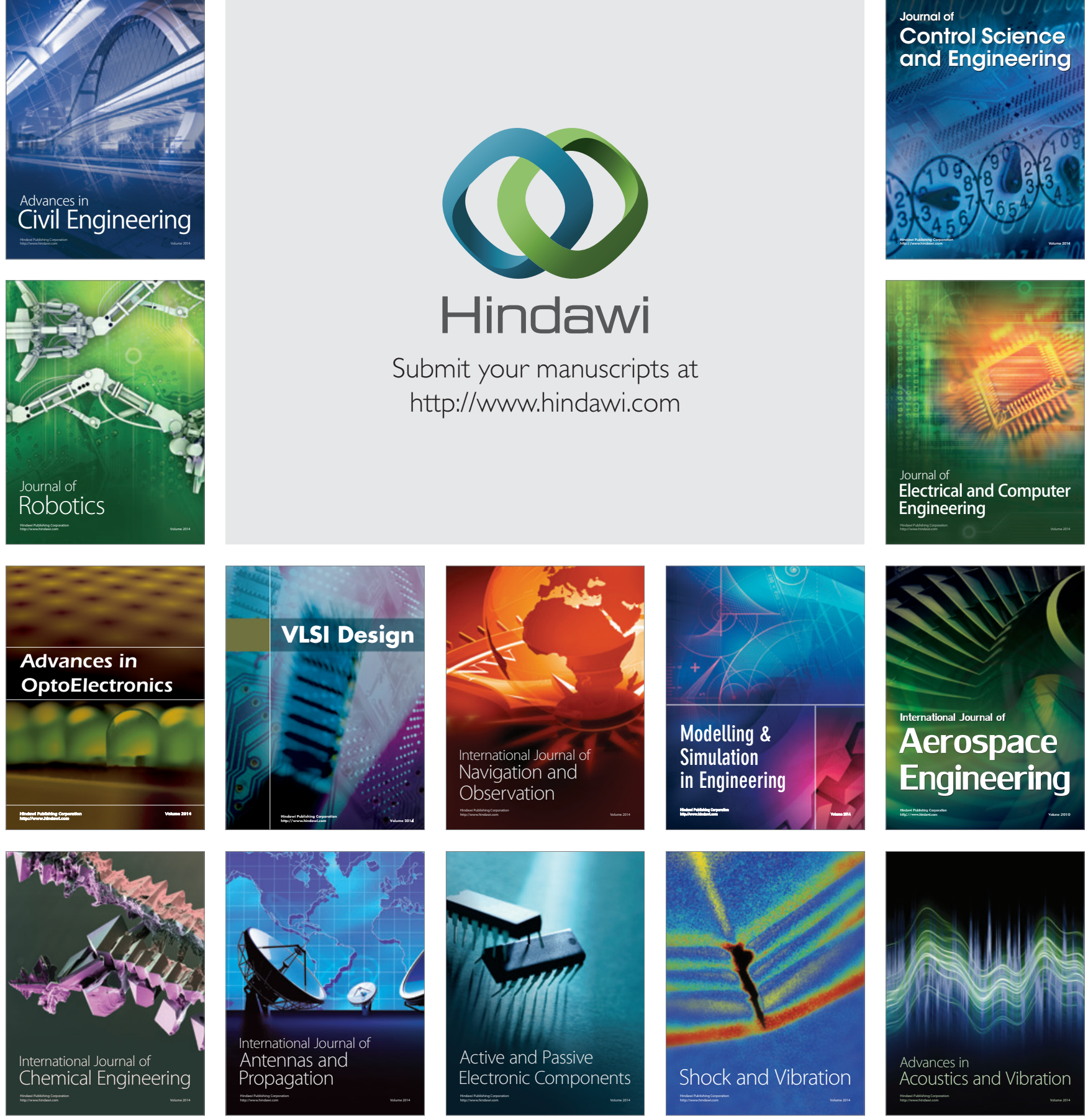